Horizons philosophiques

\title{
Notes pour une archéologie du postmodernisme
}

\section{François Rochon}

Volume 5, numéro 1, automne 1994

Esthétiques et sociétés

URI : https://id.erudit.org/iderudit/800965ar

DOI : https://doi.org/10.7202/800965ar

Aller au sommaire du numéro

Éditeur(s)

Collège Édouard-Montpetit

ISSN

1181-9227 (imprimé)

1920-2954 (numérique)

Découvrir la revue

Citer cet article

Rochon, F. (1994). Notes pour une archéologie du postmodernisme. Horizons philosophiques, 5(1), 56-73. https://doi.org/10.7202/800965ar d'utilisation que vous pouvez consulter en ligne.

https://apropos.erudit.org/fr/usagers/politique-dutilisation/ 


\section{NOTES POUR UNE ARCHÉOLOGIE DU POSTMODERNISME}

Catégorie historique, esthétique, idéologique ou simplement évaluative et normative des œuvres d'art en général et des textes littéraires en particulier? Le postmodernisme, il faut bien le reconnaître, possède une valeur heuristique qui varie beaucoup selon les types d'analyse pratiqués. D'un point de vue historique, il serait apparu vers la fin des années 1950 et au débutdes années 1960, auxÉtats-Unis et en Europe occidentale, dans la foulée d'un nouvel ordre socio-économique que le philosophe marxiste américain Fredric Jameson appelle «late capitalism ${ }^{1}$. Sur le plan philosophique, le postmodernisme marquerait la fin de ce que Jean-François Lyotard appelle l'ère des méta-récits ou grands récits de la culture occidentale moderne, - à savoirle christianisme, l'émancipation de l'homme par le moyen de la Raison, l'idéal communiste d'une société sans classe ${ }^{2}$. Enfin, de manière un peu plus précise en ce qui concerne la littérature, le postmodernisme viendrait attester de la mort du sujet, célébrée à plusieurs reprises dans la philosophie contemporaine, en révoquant la figure tutélaire de l'auteur : celui-ci, comme l'écrit Ihab Hassan dans un des premiers essais sur le postmodernisme en littérature, ne tiendrait plus alors que dans la convergence entre différentes voix qui coulent dans un texte, "a somewhat fortuitous crystallization of language ${ }^{3}$ ".

Le sens même du postmodernisme est néanmoins problématique, pour ne pas dire incertain, lorsqu'on veut en cerner de plus près la spécificité par rapport au modernisme :

1. Frederic Jameson, «Postmodernism, or The Cultural Logic of Late Capitalism", in New Left Review, no 146, 1984, p. 53-92.

2. Jean-François Lyotard, La Condition postmoderne, Paris, Minuit, 1979. Voir aussi, du même auteur, Moralités postmodeines, Paris, Galilée, 1993.

3. Ihab Hassan, The Dismemberment of Orpheus. Toward a Postmodern Literature, Madison (Wisconsin) et Londres, The University of Wisconsin Press, 1982 [1971], p. xiii. 
le postmodernisme constitue-t-il une formation discursive autonome qui tranche par rapport au modernisme? Si oui, en quoi consiste alors la singularité discursive du postmodernisme? Tient-elle de la nature même des œuvres regroupées sous ce vocable, et plus particulièrement ici des textes littéraires, ou encore de la formation de concepts et de stratégies qui relèveraient davantage des discours critiques liés au postmodernisme que des pratiques elles-mêmes? Comme l'indiquent les termes que j'emprunte ici à Michel Foucault, toute une archéologie du postmodernisme pourrait être entreprise dans la perspective de ces questions : elle permettrait de mieux comprendre l'émergence de la notion même de postmodernisme, d'en montrer la constitution comme discours en le replaçant sur le fond d'une histoire culturelle du $\mathrm{XX} \mathrm{X}^{\ominus}$ siècle, et d'en saisir les multiples enjeux (esthétique, philosophique, épistémologique). Contribuer, encore que de façon bien modeste, à cette archéologie qui reste largement à faire constitue le propos de cet article.

L'archéologie étant une méthode d'analyse des discours considérés dans leurs positivités historiques, on ne s'étonnera donc pas que le texte que je propose ici traite du passé, encore récent, plutôt que de l'immédiat et de l'actuel. Ainsi, mon analyse s'en tient aux textes critiques qui ont paru dans les années 1970 et surtout dans la première moitié des années 1980 : le propos de mon article ne consiste pas tant à effectuer un survol des théories du postmodernisme d'hier à aujourd'hui, mais plutôt à considérer la genèse - somme toute embrouillée - de ce courant de pensée qui a connu depuis un développement tout à fait prodigieux.

\section{Questions et hypothèse}

Qu'est-ce donc que le postmodernisme - et en particulier le postmodernisme en littérature - , peut-on se demander de la même manière que Foucault s'interrogeait sur ce qu'étaient la philologie, l'économie politique, la biologie dans Les Mots et les choses? Comment, alors qu'il paraît dominer aujourd'hui dans plusieurs domaines de pensée, se trouve-t-il à émerger comme discours? 
Posée de façon très générale dans les termes d'une continuité/discontinuité, la dernière question demeure toutefois encore largement débattue. Elle montre d'abord que les frontières historiques, de même que la définition du terme de postmodernisme, sont instables.

Ainsi, pour Jameson, le postmodernisme marque une nette coupure; celle-ci interviendrait vers la fin des années 1950 et au début des années 1960, et opérerait en réaction au modernisme qui dominait depuis le siècle dernier. Le postmodernisme serait donc né du rejet de l'esthétique précédente qui, si elle transgressait initialement toute une série de normes et de valeurs imposées par l'ordre culturel de la bourgeoisie, était néanmoins devenue la culture officielle au début des années 1960 : ses productions autrefois scandaleuses, comme l'écrit Hal Foster en marge du propos de Jameson, "se trouvent maintenant à l'université, dans les musées, dans les rues. Bref, comme Habermas l'écrit, le modernisme semble dominant mais mort dominant but dead ${ }^{4}$ ". Ce rejet esthétique ne serait cependant que la contrepartie superstructurelle, si l'on suit l'analyse marxiste de Jameson, d'une mutation profonde dans l'ordre socio-économique : «'émergence du postmodernisme est étroitement liée à l'émergence de cette nouvelle forme de capitalisme tardif, appelée capitalisme multinational ou de consommation ${ }^{5}$ ". Jameson reprend ici la classification de Ernest Mandel qui distingue trois phases dans le développement du capitalisme, - classification dont il se servira comme modèle pour élaborer une périodisation culturelle un peu simpliste : le réalisme au $X I X^{\ominus}$ siècle devient ainsi la contrepartie esthétique du capitalisme de marché; le modernisme de la fin du $X I X^{\ominus}$ siècle et du début du $X X^{\ominus}$, celle du capitalisme monopolistique; et le postmodernisme, celle du capitalisme multinational.

4. Hal Foster, "Postmodernism: A Preface», in The Anti-Aesthetic, Washington, Bay Presss, 1983, p. ix. Je traduis ici les propos de Foster.

5. Fredric Jameson, loc. cit., p. 125 [ma traduction]. 
Pour Jameson, donc, la coupure par laquelle le postmodernisme émerge et se pose en tant que phénomène et courant esthétiques est de nature socio-économique : l'infrastructure postindustrielle subsume globalement la culture postmoderne comme superstructure. Pour Jean-François Lyotard, qui n'adopte pas le point de vue marxiste de Jameson, la coupure sera avant tout de nature culturelle; elle n'opère pas tant depuis une mutation économique, mais plutôt depuis une pragmatique des jeux de langage dont il emprunte vaguement la notion à Wittgenstein : le terme postmoderne, écrit-il, «désigne l'état de la culture après les transformations qui ont affecté les règles des jeux de la science, de la littérature et des arts à partir de la fin du XIXe siècle ${ }^{6}$,. Force est de constater ici que la rupture qu'opère la condition postmoderne diffère grandement de celle invoquée par Jameson : historiquement, elle apparaît de façon fort paradoxale au moment même où le modernisme émerge, soit à la fin du XIXe siècle; épistémologiquement, elle ne procède pas du rejet de l'esthétique subversive du modernisme mais d'une crise de ce que Lyotard appelle les grands récits de la modernité qui sont issus de la pensée des Lumières. Cette différence qui touche au sens même du terme postmoderne tient au fait que la coupure invoquée par Jameson et Lyotard n'intervient pas, pour parler comme Foucault, sur le même objet discursif. Le modernisme (Jameson) n'est pas la modernité (Lyotard) : le premier terme désigne un courant esthétique dont Baudelaire a été un des principaux instigateurs ${ }^{7}$; le second renvoie à un projet d'émancipation de l'humanité dans les sphères des sciences et des arts, mais également dans celles du progrès technique, de la richesse et du bonheur des individus.

6. Jean-François Lyotard, La Condition postmoderne, p. 7.

7. La confusion entre les deux termes est d'autant plus grande ici quec'est sous le nom même de "modernité" que s'est développé ce courant esthétique dont Baudelaire, avec un texte intitulé "Le peintre de la vie moderne", a été le principal instigateur en France. Cf. : Charles Baudelaire, Écrits esthétiques, Paris, UGE, 1986 [1863], p. $360-404$. 
Dans un livre paru il y a une dizaine d'années, Literary History, Modernism, and Postmodernism, Douwe Fokkema privilégie l'approche sémiotique pour départager le modernisme du postmodernisme dans le champ plus particulier de la littérature. La notion de code est l'outil conceptuel retenu : Fokkema distingue cinq types de code, qui opèrent dans un texte littéraire, - allant ainsi du plus global, comme le code linguistique, au plus restreint, comme l'idiolecte de l'auteur, en passant par les codes littéraires (grâce auxquels un texte signale son appartenance au champ de la littérature), le code générique (roman, poésie, théâtre, ...) et le period code ou group code (à savoir les différents mouvements et écoles qui constituent l'histoire littéraire). En montrant qu'elle relève de ce dernier code, Fokkema retrace la coupure entre le modernisme et le postmodernisme au moyen d'un système d'oppositions : le sujet moderne représenterait ainsi le monde d'un point de vue personnel, tandis que le sujet postmoderne aurait renoncé à une représentation du monde élaborée en fonction des convictions et de la sensibilité d'un sujet individuel; le récit moderne est présenté comme jamais fini, à la limite interminable, tandis que le récit postmoderne, outre qu'il puisse s'interrompre de façon abrupte, exploite le procédé des fins multiples, faisant du texte une structure labyrinthique dont Borges nous a donné le modèle; alors que le modernisme rejetait toute explication de la réalité, le postmodernisme renonce à la tentative même d'expliquer; le rôle du lecteur est plus accentué dans l'esthétique postmoderniste que dans le modernisme; enfin, alors que les textes du modernisme représentaient des mondes possibles, concevables dans la réalité, les récits postmodernistes représentent des mondes qu'on ne peut concevoir autrement que dans l'imagination ${ }^{8}$.

Pour intéressante qu'elle soit, la tentative de Fokkema est cependant emblématique d'une difficulté théorique à laquelle se heurtent la plupart des études qui veulent cerner la spécificité

8. Cf.: Douwe W. Fokkema, Literary History, Modernism, and Postmodemism, Amsterdam et Phidadelphia, John Benjamins Company, 1984, p. 37-56. 
du postmodernisme en littérature : le système d'oppositions qu'il élabore pour départager le postmodernisme du modernisme est davantage une question de degrés et de nuances entre ces deux esthétiques que de rupture et de contrastes. Je ne donnerai ici qu'un exemple prouvant cet état de fait. Le rôle du lecteur ainsi que le caractère imprévisible du récit sont certes des constantes de textes dits postmodernistes, comme dans $\mathrm{Si}$ par une nuit d'hiver un voyageurd'Italo Calvino, pour donner un cas exemplaire; mais ces traits formels se retrouvent de façon tout aussi insistante dans certains textes modernes comme Paludes de Gide, et même au XVIII ${ }^{e}$ siècle avec Tristram Shandy de Sterne et Jacques le fataliste de Diderot. Chez ce dernier, comme on sait, le rôle dévolu à un lecteur fictif et le caractère fragmenté, constamment interrompu et en bout de piste indéterminé des anecdoctes racontées dans Jacques le fataliste participent même, sous la forme d'un paradoxe qui parcourt tout le texte, de l'argument majeur de tout le roman, à savoir la thèse du cours nécessaire des événements.

Reconnue à maintes reprises, enjeu de nombreux débats qui encore aujourd'hui vont jusqu'à remettre en question l'existence même du postmodernisme, cette difficulté théorique constitue la tâche la plus pressante du projet d'une archéologie du postmodernisme en littérature. L'étude de ce courant littéraire à l'instar d'une formation discursive ferait en quelque sorte entorse à la méthode de Foucault: le postmodernisme reprenant le programme esthétique du modernisme, bien plus qu'il ne s'y oppose $^{9}$, les notions de coupure et de rupture perdent ainsi de leur valeur descriptive. Les textes modernistes, comme ceux de Gide, de Proust, de Joyce et de Virginia Woolf, rejetaient d'emblée l'esthétique de la période qui les précédait, à savoir le

9. C'est là l'opinion qu'avancent certains critiques américains, et qui semble se confirmer ici. Pour Gerald Graff, par exemple, la coupure qu'opérerait le postmodernisme en littérature n'est qu'un mythe (cf. "The Myth of The Postmodernist Breakthrough", in Tri-Quaterly, $n^{\circ}$ 26). Pour Graff, rapporte John Barth, "la fiction postmoderniste ne fait que porter un peu plus loin - à son aboutissement logique mais discutable - le programme antirationaliste, anti-réaliste, anti-bourgeois du modernisme, mais sans adversaire solide (...) ni amarrage solide dans le réalisme quotidien contre lequel il s'élève». Cf.: John Barth, "La littérature de renouvellement : la fiction postmoderniste", in Poétique, $n^{\circ}$ 48, 1981, p. 400. 
réalisme et le naturalisme. Contrairement à ce que peut écrire Jameson, les textes censés appartenir au postmodernisme, ceux de Borges et de Calvino notamment, n'opèrent pas un rejet radical de la tradition littéraire qui les précède; ils ne manifestent pas le désir - comme c'était le cas, ainsi que l'explique Paul de Man, du courant moderniste - «de se débarrasser de ce qui existait auparavant, dans l'espoir de parvenir finalementà un point qu'on pourrait appeler un véritable présent, un point d'origine qui marque un nouveau commencement ${ }^{10}$ ". Ils opèrent plutôt à l'intérieur même de cette tradition, ils en redistribuent les divers courants qui interviennent dans le récit postmoderne sous forme de parodies et de pastiches, mais également de principes d'organisation thématiques, rhétoriques et sémiotiques. Certains textes de Calvino sont emblématiques de cette redistribution. Dans Le Château des destins croisés, un jeu de tarots disposés sur une table permetà divers personnages, parmi lesquels on reconnaît l'Orlando innamorato de Boiardo (fin du XV ${ }^{\ominus}$ siècle), Hamlet, Hélène de Troie, etc., de raconter leurs histoires. Dans Si par une nuit d'hiver un voyageur, la représentation de l'activité de la lecture opère une redistribution fragmentée des principales tendances selon lesquelles la forme romanesque a évolué depuis Don Quichotte : on trouve ainsi, dans cet ouvrage de Calvino, un roman réaliste qui débute dans une gare italienne; un roman engagé; un roman policier qui se situe en Amérique du Sud; un roman métaphysique dans lequel de multiples miroirs viennent remettre en question l'identité d'un narrateur mégalomane; un roman érotique dont l'action se passe dans un jardin japonais, etc.; sans oublier le roman intermittent du Lecteur du roman de Calvino, structure d'engendrement de ce texte souvent considéré comme un prototype de l'œuvre postmoderne.

10. Paul de Man, Blindness and Insight, Minneapolis, University of Minnesota Press, 1983 [1971], p. 148 [ma traduction]. 
Cette opération de redistribution de la tradition littéraire existante soulève cependant un des enjeux les plus problématiques du postmodernisme, celui de l'invention. Comment inventer, comment créer encore du nouveau en littérature? Le postmodernisme n'est-il donc qu'une réinscription ludique du programme anti-réaliste du modernisme? Pour Jameson, le postmodernisme n'est pas une esthétique de l'innovation tout aurait été inventé - , mais tout au contraire de la répétition, du simulacre, jeu et masque conjugués ${ }^{11}$.

Or, ce que Jameson considère comme un "échec nécessaire" constitue tout aussi bien un renouvellement esthétique, - ou plus justement un déplacement de certains impératifs esthétiques, notamment celui du caractère représentatif de l'art en général et de la littérature en particulier. À la lecture du texte de Jameson paru dans The Anti-Aesthetic, on s'avise que cet échec correspond à une crise aigüe de la représentation : défini comme l'un des principaux traits du postmodernisme, le pastiche constitue une imitation dont l'objet n'est d'aucune manière référentiel mais purement discursif. En outre, cette crise se trouve aggravée par la mort du sujet, ce que Jameson appelle en anglais "the end of individualism as such", - le je étant passé d'un statut psychologique, qui circonscrivait son identité, à un statut purement linguistique, forme vide de discours. Ce déplacement esthétique nous amène donc à formuler et à examiner l'hypothèse suivante, quitte à l'invalider par la suite : après le réalisme et le naturalisme dont le programme consistait grosso modo dans la représentation de la réalité; après le modernisme qui rejetait ce programme pour représenter ce qu'on pourrait appeler les derniers avatars du moi conçu comme sujet psychologique individuel; le

11. "Here, once again, pastiche : in a world in which stylistic innovation is no longer possible, all that is left is to imitate dead styles, to speak through the masks and with the voices of the styles of the imaginary museum. But this means that contemporary or postmodemist art is going to be about art itself in a new kind of way; even more, it means that one of its essential messages will involve the necessary failure of art and the aesthetic, the failure of the new, the imprisonment in the past». Fredric Jameson, "Postmodernism and Consumer Society", in Hal Foster (éd.), The AntiAesthetic, p. 115-116. 
postmodernisme se caractériserait principalement par une représentation de discours où opèrent divers régimes de jeux de langage. Ces derniers seraient au nombre de trois : un régime d'ordre déictique, qui diffracte le moi en une série de positions d'énonciation irréductibles à un individu; un régime d'ordre narratif, qui expose le récit postmoderne comme un ensemble de stratégies textuelles avouées, - jeux de mots, mise en abyme, représentation du rôle du lecteur, métanarration, etc.; et un régime d'ordre épistémique, qui intervient dans le récit sous forme de fragments narrativisés de savoirs, d'idées et de motifs empruntés à différents modèles scientifiques contemporains.

\section{Problèmes du postmodernisme littéraire}

1. Le sujet et la représentation. "Où maintenant? Quand maintenant? Qui maintenant ${ }^{12}$ »? Ces questions sur lesquelles s'ouvre L'Innommable de Beckett circonscrivent trois des principales catégories de la représentation littéraire : l'espace, le temps et le sujet. Or, ces questions demeureront sans réponse dans la suite du roman. L'espace se trouve ici dépourvu de toutes frontières, à la mesure d'un trajet sans fin dont on ne connaît ni le lieu de départ ni le lieu d'arrivée : «L'essentiel est que je n'arrive jamais nulle part, que je ne sois nulle part, ni chez Mahood, ni chez Worm, ni chez moì (p. 86). Le temps est en outre privé de sa dimension linéaire, il est également sans début ni fin : "sans l'illusion d'avoir commencé un jour, de pouvoir un jour conclure, mais c'est trop difficile, dépourvu de but, de ne pas se vouloir une fin, de raison d'être, un temps où l'on n'était pas» (p. 163). Enfin, on assiste non pas à la mort du sujet mais à l'agonie sans rémission d'un je qui n'en finit plus de mourir, toujours pressé par le devoir de parler, de se dire et l'impossibilité de le faire : «il faut continuer, je ne peux pas continuer, il faut continuer, je vais donc continuer, il faut dire des mots, tant qu'il y en a, il faut les dire, jusqu'à ce qu'ils me trouvent, jusqu'à ce

12. Samuel Beckett, L'Innommable, Paris, Minuit, 1953, p. 7. Toutes les citations contenues dans ce paragraphe proviennent de cet ouvrage; le numéro de page est indiqué entre parenthèses. 
qu'ils me disent, étrange peine, étrange faute» (p. 213). En fait, il ne reste plus de l'esthétique traditionnelle de la représentation que la mise en forme bégayante d'un discours, d'une voix sans lieu ni âge, "grosse boule parlante" qui dit l'impossibilité de la représentation. Ou plus précisément encore, qui représente cette impossibilité même :

\begin{abstract}
Le fait semble être, si dans la situation où je suis on peut parler de faits, non seulement que je vais avoir à parler de choses dont je ne peux parler, mais encore, ce qui est encore plus intéressant, que je, ce qui est encore plus intéressant, que je, je ne sais plus, ça ne fait rien. Cependant, je suis obligé de parler. Je ne me tairai jamais. Jamais. (p. 8)
\end{abstract}

Beckett constitue en quelque sorte ce que la rhétorique de l'Antiquité appelait un imago, ou figure exemplaire, pour le postmodernisme : pour I. Hassan, Beckett est un "exemple suprême d'artiste postmoderne ${ }^{13^{3}}$; ; pour Jameson également, il est "l'un des artistes postmodernes les plus significatifs ${ }^{14}$ ". La crise de la représentation dont L'Innommable est la mise en scène s'accorde bien avec la définition d'ordre esthétique - et non plus multi-catégorielle comme dans La Condition postmoderne - que J.-F. Lyotard donne du postmoderne dans un article publié dans un livre au titre humoristique : Le Postmoderne expliqué aux enfants. Lyotard y reprend la catégorie du sublime telle que pensée par Kant dans la Critique de la faculté de juger. Kant distingue dans cet ouvrage le jugement de goût et le sublime. Dans le premier, un accord intervient entre les facultés du sujet, principalement la faculté de concevoir, et celles qui consistent à «présenter un objet correspondant au concept ${ }^{15}$ ", ce qui procure un sentiment de satisfaction. Au contraire, le sublime résulte d'un conflit entre ces deux facultés : il a lieu, écrit Lyotard en marge de Kant, quand «l'imagination échoue à présenter un objet qui vienne, ne serait-ce qu'en principe, s'accorder avec un concept". Dans

13. Ihab Hassan, op. cit., p. 210 [ma traduction].

14. Fredric Jameson, loc. cit., p. 118 [ma traduction].

15. Jean-François Lyotard, Le Postmoderne expliqué aux enfants, Paris, Galilée, 1986, p. 26. 
L'Innommable, l'objet sur lequel porte cet échec est en fait le sujet lui-même, dépositaire de la re-présentation : le conflit intervient entre l'idée cartésienne de sujet qui domine encore et sa présentation devenue impossible: «Dire je. Sans le penser ${ }^{15}$ ".

Or, cette crise de la représentation qui affecte le sujet était déjà donnée à lire dans plusieurs textes modernes. Qu'on pense seulement ici à l'œuvre de Virginia Woolf : dans The Waves, l'instance de narration se trouve diffractée en six voix, - diffractée dans son sens physique, le sujet fragmenté de l'énonciation étant devenu le mouvement ondulatoire de l'eau : "so fluid has my body become, even at the touch of a finger into one full drop, which fills itself, which quivers, which flashes, which falls in ectasy ${ }^{16}$ ". La représentation impossible du sujet en tant qu'individu portant un nom et une identité bien définie correspond bien ici au sublime tel que pensé par Kant : elle est à la fois un "sentiment de peine», suscité par l'impossibilité à laquelle se heurte le sujet de répondre à une logique de l'identité, - "Identity failed me. We are nothing, I said, and fell ${ }^{17}$ »; et un "sentiment de joie" ou ectasy, par lequel le sujet déborde largement la notion d'individu et devient ainsi un sujet multiple ou transsujet, - «I am not one person; I am many people; I do not altogether know who I am ${ }^{18}$ ".

L'esthétique postmoderne ne trouve donc pas dans la catégorie du sublime kantien un de ses traits caractéristiques. Lyotard invite d'ailleurs lui-même à une telle remarque lorsqu'il écrit : «Le postmoderne serait ce qui dans le moderne allègue l'imprésentable dans la présentation elle-même ${ }^{19}$ ". Défini de la sorte, le postmoderne ne renvoie pas à une quelconque période de l'histoire littéraire, - ce que Fokkema appelle le period code; il n'indique pas un développement qui serait ultérieur au modernisme (ce que le préfixe "post» annonce pourtant), ni même à la modernité puisque la notion du sublime kantien

16. Samuel Beckett, op. cit., p. 7.

17. Virginia Woolf, The Waves, Londres, Granada, 1977 [1931], p. 149.

18. Ibid., p. 43.

19. Ibid., p. 187.

20. Jean-François Lyotard, op. cit., p. 32. 
apparaissait à la même époque que le projet des Lumières prenait forme. Le postmoderne tel que défini par Lyotard constituerait de façon fort paradoxale une catégorie transhistorique, - «Une œuvre ne peut devenir moderne que si elle est d'abord postmoderne ${ }^{21}$, écrit encore Lyotard. Or, ce paradoxe qui ressortità la dimension historique du postmoderne n'est pas vraiment nouveau : ce n'est en fait qu'une répétition contemporaine - à deux termes près - du paradoxe qu'analysait Paul de Man à propos de la modernité et de son esthétique: "Si l'histoire ne devient pas simple régression ou paralysie, c'est qu'elle compte sur la modernité pour se poursuivre et pour se renouveler (for its duration and renewal); mais la modernité ne peut s'affirmer sans parfois être absorbée et réintégrée dans un processus historique régressif ${ }^{22}$ ". Postmoderne remplacerait ici moderne, et modernité prend la place de histoire : ainsi, la modernité serait devenue, dans l'optique du paradoxe énoncé par Lyotard, ce processus ou mouvement régressif de l'histoire auquel elle résisterait cependant et voudrait échapper sous le nom de postmoderne.

2. Le texte et ses miroirs. La plupart des critiques qui ont étudié le postmodernisme dans sa composante littéraire ont porté leur attention sur le plan formel, à savoir les stratégies textuelles qui gouvernent un récit. Ces stratégies ressortissent à divers niveaux narratifs : l'énonciation (inscription du narrateur et du narrataire dans le récit), diégèse (thèmes de l'écriture comme projet et acte de la rupture), codes (procédés relatifs à l'autoreprésentation et à l'intertextualité, jeux sur le signifiant, etc. ${ }^{23}$. Or, ces stratégies n'apparaissent pas avec le roman postmoderne; elles opéraient déjà dans le roman moderne, chez Gide notamment, à qui l'on doit la notion de mise en abyme; dans le roman traditionnel, - Robinson Crusoe (1719)

21. Ibid., p. 30.

22. Paul de Man, op. cit., p. 151 [ma traduction].

23. Pour un aperçu plus complet et plus détaillé de ces stratégies textuelles dans le cadre du roman postmoderne, voir : Janet M. Paterson, "Le Roman "postmoderne" : mise au point et perspectives", in Canadian Review of Comparative Literature/ Revue canadienne de littérature comparée, juin 1986, p. 238-254. 
mettait déjà en scène un narrateur à la première personne, Tristram Shandy (1760-1767) dialoguait avec son lecteur et laissait des pages blanches sur lesquelles il lui demandait d'écrire ce qui lui passait par la tête; et jusqu'aux origines du roman occidental, - le fameux Don Quichotte (1605-1615) de Cervantes se trouvant déjà à diffracter l'instance de narration dans la mesure où le narrateur anonyme du «Prologue» du roman ne fait que rapporter le récit traduit de Quichotte, récit dont l'auteur véritable serait l'historien arabe et cependant menteurCid HametBen-Engeli. La critique adopte deux attitudes devant cet état de fait. La première consiste à reconnaître des précurseurs du postmodernisme, ce que John Barth appelle "les premières figures anticipant "l'esthétique littéraire postmoderniste» 24 ". Or, cet argument contrevient encore une fois à l'idée voulant que le postmodernisme marque une coupure par rapport aux esthétiques littéraires précédentes; il montrerait au contraire que le postmodernisme s'inscrit tout à fait dans le développement de la littérature occidentale, tel une extension du modernisme, - ce que nie Barth avec énergie sans préciser toutefois ce qui distingue le postmodernisme des esthétiques précédentes. La seconde attitude critique n'est pas éloignée de la première, bien que sa valeur heuristique soit plus facilement cernable : elle voit dans le postmodernisme un surcodage, comme dit J. M. Paterson, et une extension des stratégies textuelles opérant déjà dans une mesure moindre dans les romans de types moderne et traditionnel.

À première vue, pareille attitude ne ferait que confirmer que, sur le plan formel, la spécificité du postmodernisme tient davantage dans une question de degrés que de rupture par rapport aux esthétiques précédentes. Or, le surcodage de procédés littéraires qui intervient tant au niveau de l'énonciation que du code vient exposer le récit postmoderne telle une structure narrative opérant divers jeux de langage. Le texte prend ainsi une fonction ludique de toute première importance, qui subsume en quelque sorte les fonctions linguistiques du 
schéma jakobsonien de la communication. La fonction référentielle est en grande partie contenue dans la fonction métalinguistique, on pourrait dire ici métanarrative, - le texte est tout occupé à représenter ses propres processus d'engendrement, ce qui donne lieu à une emphatic selfreflexiveness pour parler comme C. Russell ${ }^{25}$. La fonction poétique sert à produire de nombreux jeux de mots, - qu'on pense ici aux romans de Boris Vian qu'on oublie souvent mais quiseraient postmodernes à plus d'un titre, en raison précisément de leur caractère ludique et parodique. Enfin, les fonctions phatique et conative développent le rôle du lecteur, - le texte représente non seulement ses processus d'écriture mais également l'activité même de sa lecture, comme c'est le cas au tout début de Si par une nuit d'hiver un voyageur: "Tu vas commencer le nouveau roman d'Italo Calvino, Si par une nuit d'hiver un voyageur ${ }^{26}$ ".

Dans la mesure où elle domine sur les diverses fonctions du langage répertoriées par Jakobson, cette fonction ludique introduit sur le plan formel une certaine distinction entre le modernisme et le postmodernisme. Le programme antirationaliste, anti-réaliste et anti-bourgeois par lequel on définit généralement le modernisme avait tendance à oblitérer la dimension ludique des textes littéraires: les stratégies narratives qui accentuaient le caractère autoreprésentatif de ces textes prenaient en soi et d'abord une valeur subversive par rapport à l'esthétique qui dominait précédemment, à savoir le réalisme et le naturalisme. À l'inverse, le postmodernisme redonnerait à ces stratégies leur dimension ludique première : celles-ci ne constituent pas une subversion de l'esthétique moderniste, ils ne transgressent pas son programme anti-rationaliste et antiréaliste; au contraire, elles le poursuivent mais en l'affirmant, en le radicalisant en quelque sorte sur le mode ludique.

25. Charles Russell, "The Context of The Concept", in Harry R. Gravin, Romanticism, Modernism, Postmodernism, Londres et Toronto, Bucknell University Press, 1980, p. 183.

26. Italo Calvino, Si par une nuit d'hiver un voyageur, Paris, Seuil, 1981, p. 7. 
3. Science et fiction. Le surcodage de procédés littéraires amène le roman postmoderne à produire et à organiser ses propres réseaux de sens, à dévoiler son fonctionnement narratif et à élaborer son propre contexte d'interprétation. Cette exploration du texte par lui-même opère souvent depuis une projection de modèles scientifiques contemporains dans le cadre de la fiction : ceux-ci interviennent alors sous forme de représentations d'action et de personnages, de thèmes et de motifs, de figures de rhétorique ou encore de systèmes tout faits de causalité, qui opèrent tant au niveau des structures narratives d'un texte qu'à celui de la matière même de son intrigue.

De tous les modèles scientifiques du discours desquels on peut trouver trace dans des textes littéraires dits postmodernes, c'est sans doute la cybernétique qui intervient de la façon la plus insistante et la plus prégnante. La "fiction cybernétique», comme l'appelle David Porush, constituerait du reste une souscatégorie de la fiction postmoderne : elle comprendrait des textes qui «imitent eux-mêmes des machines, se donnent pour des machines ou se trouvent "générés" par des machines, ou encore sont structurés comme des mécanismes très bien rodés ${ }^{27}$ ". Porush compte parmi les récits qui entrent dans cette catégorie ceux de John Barth, Donald Barthelme, Samuel Beckett, William Burroughs, Kurt Vonnegut, Thoman Pynchon, etc. Je ne prendrai ici qu'un exemple, dont j'ai déjà parlé, pour montrer comment la cybernétique peut structurer un roman : $S i$ par une nuit d'hiver un voyageur de Calvino.

Construit analogiquement sous l'espèce d'une machine, le roman de Calvino expose de fait son programme diégétique, ses opérations et ses stratégies de narration en termes de redondance et de bruit, d'ordre et de hasard, d'entropie et de néguentropie, - notions qui sont toutes au cœur de la cybernétique. Dès le premier chapitre, le roman représente ainsi l'activité de sa propre lecture, ses conditions physiques aussi bien que psychologiques : il met en scène un Lecteur fictif,

27. David Porush, "Technology and Postmodernism : Cybernetic Fiction", in Substance, $\mathrm{n}^{\circ} 27,1980$, p. 83 [ma traduction]. Cf. aussi du même auteur : The Soft Machine. Cybernetic Fiction, New York, Methuen, 1985. 
personnage principal de l'ouvrage, qui est sur le point de lire le dernier roman de Calvino, celui-là même qu'annonçait le titre du livre que l'on tient dans nos mains. Opère ici une disjonction narrative qui donne toute la structure éminemment ludique du roman de Calvino : le roman que lit le lecteur réel n'est pas le même que celui que va entreprendre le Lecteur fictif; il en est plutôt la représentation. Ainsi, quand Siparune nuitcommence, nous ne lisons pas ce que le personnage du Lecteur lit, mais plutôt la représentation du roman lui-même en train de commencer :

Le roman commence dans une gare de chemin de fer, une locomotive souffle, un sifflement de piston couvre l'ouverture du chapitre, un nuage de fumée cache en partie le premier alinéa. Dans l'odeur de gare passe une bouffée d'odeur de buffet. Quelqu'un regarde à travers les vitres embuées, ouvre la porte vitrée du bar, tout est brumeux à l'intérieur, comme vu à travers des yeux de myope [...]. Ce sont les pages du livre qui sont embuées, comme les vitres d'un vieux train; c'est sur les phrases que se pose le nuage de fumée ${ }^{28}$.

Assimilé métaphoriquement à une locomotive du siècle dernier, le roman devient en quelque sorte une mécanique langagière soumise aux processus de transformation de l'énergie : l'insistance mise sur le sème de fumée, qui constitue un paradigme dans l'extrait cité, suggère bien du reste que le texte se construit à l'instar d'une force de travail. Intervenant sur le langage pour remédier peu à peu à son inertie, au caractère encore indéterminé d'un récit qui vient à peine de commencer, cette force se trouve convertie en chaleur pour produire du mouvement : c'est-à-dire, analogiquement ici, du texte comportant des personnages et une intrigue. Or, après une trentaine de pages, au moment même où le Lecteur fictif commence à se passionner pour l'histoire, le récit tombe dans un état de désordre : la suite ne fait que répéter ce que le Lecteur vient de lire. C'est qu'une erreur d'imprimerie s'est

28. Italo Calvino, op. cit., p. 15. Les citations dont la référence paginale sera donnée dans le corps du texte, entre parenthèses, proviennent de ce livre. 
glissée dans l'ouvrage : "On a relié ensemble des exemplaires d'un seul cahier, il n'y a plus une seule bonne page dans tout le cahier". (p. 30) Parfaitement redondant pour le Lecteur fictif, le roman "se dégrade enfin dans un tourbillon d'entropie»; sa quantité d'information est alors réduite à un ensemble indéchiffrable de "redondances et de bruits", comme le précise encore le narrateur. Or, il en va autement pour le lecteur réel que nous sommes : celui-ci n'est pas confronté à une dégradation d'énergie textuelle mais à une quantité additive d'information : en laissant le Lecteur fictif «à la merci du hasard, de l'aléatoire, de la probabilité" (p. 31), le roman se trouve à transformer son entropie constitutive en une entropie négative ou néguentropie, qui opère un nouveau jeu de narration. De fait, l'histoire redondante que litle Lecteurfictiffait placeàl'histoire imprévisible de ce Lecteur qui va à la librairie échanger son exemplaire, qui y rencontre une Lectrice dont il tombe amoureux, et qui toujours commence à lire des romans sans jamais pouvoir en connaître la fin.

Cet article, on l'aura deviné, demeure prospectif. Son objectif consistaità exposer, sans prétendre à quelque exhaustivité que ce soit, la diversité des types d'analyse qui traitent du postmodernisme. Or, cette diversité intéresse au plus haut point le projet d'une archéologie du postmodernisme dans le champ plus circonscrit de la littérature. Elle indique en premier lieu que ce terme critique, qui a une valeur heuristique certaine, ne renvoie cependant pas toujours au même objet discursif. En ce sens, la prise en compte du postmodernisme littéraire à l'instar d'une formation discursive autonome opérerait un déplacement théorique de taille : une archéologie du postmodernisme devrait porter tout autant sur les textes littéraires qu'on dit appartenir à ce courant, que sur les discours critiques qui veulent cerner la spécificité même de ce courant sur le plan esthétique. Qu'est-ce donc que le postmodernisme, conçu à la fois comme un ensemble de textes littéraires et comme catégorie de la critique contemporaine? Ce travail est, à ma connaissance, peu entrepris; il y a quelques années, Michaël La Chance l'amorçait sans doute dans un article au titre fort suggestif : "Le 
postmodernisme est-il généré par la critique"? À cette question, La Chance répondait par l'affirmative, allant même jusqu'à dire que «la critique postmoderniste ne saura rendre compte des expériences artistiques que pour autant qu'elle aura été constitutive de celles-ci ${ }^{29}$ ".

En second lieu, le projet d'une archéologie du postmodernisme en littérature montre que les notions de coupure et de rupture perdent quelque peu de la valeur descriptive que leur assignait Foucault dans L'Archéologie du savoir. Le postmodernisme, pas plus d'ailleurs que le modernisme et le réalisme auxquels il emprunte, et dont il redistribue ou encore parodie certaines de leurs stratégies textuelles, n'est un courant esthétique pleinement autonome; il ne constitue pas une formation discursive homogène, stable et bien découpée du point de vue historique et épistémologique. Ce serait plutôt dans sa nature, pourrait-on dire, d'être hétérogène, toujours un peu déplacé par rapport à lui-même et encore peu cohérent. De fait, qu'ont en commun les textes dépouillés de Beckett et ceux ludiques de Calvino? Les récits fantastiques de Garcia Marquez, qui puisent abondamment dans la tradition orale latinoaméricaine, et les romans formalistes de Robbe-Grilet, qui constituent dans leurs limites extrêmes une entreprise d'asémie de la littérature? Enfin, dernière question qui les intègre peutêtre toutes, comment décrire et penser cette hétérogénéité discursive sous le nom unifié de postmodernisme sans galvauder le sens même de ce terme?

François Rochon Cégep de Saint-Laurent

29. Michä̈l La Chance, "Le postmodernisme est-il généré par la critique" ? in La Petite revue de philosophie, vol. 9, n 1, automne 1987, p. 105. 